\title{
Papers to appear in forthcoming issues
}

Aliste Prieto, J.

Allouche, J.-P., Clarke, M. and Sidorov, $\mathrm{N}$.

Austin, T.

Baladi, V. and Smania, D.

Banyaga, A. and Hurtubise, D. E.

Barral, J. and Seuret, S.

Barwell, A., Good, C., Knight, R. and Raines, B. E.

Bautista, S. and Morales, C.

Bayart, F., Costakis, G. and Hadjiloucas, D.

Begun, B. and del Junco, A.

Bergelson, V. and Håland Knutson, I. J.

Bezuglyi, S., Kwiatkowski, J., Medynets, K. and Solomyak, B.

Blokh, A., Buff, X., Chéritat, A. and Oversteegen, L.

Bonatti, Ch., Gómez-Mont, X. and Vila-Freyer, R.

Bourgain, J., Lindenstrauss, E., Michel, P. and Venkatesh, A.

Bowen, L.

Boyland, P.

Bressaud, X., Durand, F. and Maass, A.

Bressaud, X., Hubert, P. and Maass, A.

Breuillard, E.

Bruin, H., Demers, M. and Melbourne, I.
Translation numbers for a class of maps on the dynamical systems arising from quasicrystals in the real line

Periodic unique beta-expansions: the Sharkovskiĭ ordering

On the norm convergence of non-conventional ergodic averages

Alternative proofs of linear response for piecewise expanding unimodal maps

The Morse-Bott inequalities via a dynamical systems approach

The singularity spectrum of the inverse of cookie-cutters

A characterization of $\omega$-limit sets in shift spaces

A sectional-Anosov connecting lemma

Topologically transitive skew-products of operators

Partitions with independent iterates in random dynamical systems

Weak mixing implies weak mixing of higher orders along tempered functions

Invariant measures on stationary Bratteli diagrams

The solar Julia sets of basic quadratic Cremer polynomials

Statistical behaviour of the leaves of Riccati foliations

Some effective results for $\times a \times b$

Invariant measures on the space of horofunctions of a word hyperbolic group

Transitivity of surface dynamics lifted to Abelian covers

On the eigenvalues of finite rank Bratteli-Vershik dynamical systems

Persistence of wandering intervals in self-similar affine interval exchange transformations

Equidistribution of dense subgroups on nilpotent Lie groups

Existence and convergence properties of physical measures for certain dynamical systems with holes 
Buzzi, J.

Calta, K. and Wortman, K.

Carvalho, A. N., Langa, J. A. and Robinson, J. C.

Cellarosi, F.

Chazottes, J.-R., Collet, P., Redig, F. and Verbitskiy, E.

Cuny, C.

Dajani, K., Hartono, Y. and Kraaikamp, C.

de la Llave, R. and Windsor, A.

Díaz, L. J. and Gorodetski, A.

Dooley, A. H. and

Golodets, V. Ya.

dos Santos, N. M. and Urzúa-Luz, R.

Dutkay, D. E., Jorgensen, P. E. T. and Picioroaga, G.

Einsiedler, M. and Fish, A.

Elekes, M., Keleti, T. and Máthé, A.

Faller, B. and Pfister, C.-E.

Fenley, S., Feres, R. and Parwani, K.

Froyland, G., Lloyd, S. and Quas, A.

Gogolev, A.

Góra, P.

Guilloux, A.

Gupta, C.

Gutkin, E. and Rams, M.

Hayashi, S.

Hochman, M.
Maximal entropy measures for piecewise affine surface homeomorphisms

On unipotent flows in $\mathcal{H}(1,1)$

Lower semicontinuity of attractors for non-autonomous dynamical systems

Renewal-type limit theorem for continued fractions with even partial quotients

A concentration inequality for interval maps with an indifferent fixed point

The a.s. convergence of the one-sided ergodic Hilbert transform

Mixing properties of $(\alpha, \beta)$-expansions

Livšic theorems for non-commutative groups including diffeomorphism groups and results on the existence of conformal structures for Anosov systems

Non-hyperbolic ergodic measures for non-hyperbolic homoclinic classes

The geometric dimension of an equivalence relation and finite extensions of countable groups

Minimal homeomorphisms on low-dimension tori

Unitary representations of wavelet groups and encoding of iterated function systems in solenoids

Rigidity of measures invariant under the action of a multiplicative semigroup of polynomial growth on $\mathbb{T}$

Self-similar and self-affine sets; measure of the intersection of two copies

A point is normal for almost all maps $\beta x+\alpha \bmod 1$ or generalized $\beta$-transformations

Harmonic functions on $\mathbb{R}$-covered foliations

Coherent structures and isolated spectrum for Perron-Frobenius cocycles

On diffeomorphisms Hölder conjugate to Anosov ones

Invariant densities for piecewise linear maps of the unit interval

A brief remark on orbits of $\operatorname{SL}(2, \mathbb{Z})$ in the euclidean plane

Extreme value distributions for some classes of non-uniformly partially hyperbolic dynamical systems

Growth rates for geometric complexities and counting functions in polygonal billiards

An extension of the ergodic closing lemma

On the automorphism groups of multidimensional shifts of finite type 
$\mathrm{Hu}, \mathrm{H}$. and Vaienti, S.

Huang, W., Ye, X. and Zhang, G.

Huveneers, F.

Ingle, W., Kaufmann, J. and Wolf, C.

Iommi, G.

Ito, $\mathrm{H}$.

Ji, L. and Weber, A.

Jiang, Y. and Ye, Y.-L.

Julien, A.

Jung, U.

Kanai, M.

Kennedy, J., Raines, B. E. and Stockman, D. R.

Kittrell, J. and Tsankov, T.

Koch, H. and Kocić, S.

Kohlenbach, U. and Leuştean, L.

Leibman, A.

Leplaideur, R. and Rios, I.

Levin, G. and Świątek, G.

Liu, C.

Massart, D.

Melbourne, I., Niţică, V. and Török, A.

Morris, I. D.

Nayak, T. and Prasad, M. G. P.

Osipenko, G.

Pask, D., Raeburn, I. and Weaver, N. A.

Pikuła, R.

Pries, C.

Quas, A. and Wierdl, M.
Absolutely continuous invariant measures for non-uniformly expanding maps

Lowering topological entropy over subsets

Subdiffusive behavior generated by irrational rotations

Natural invariant measures, divergence points and dimension in one-dimensional holomorphic dynamics

Multifractal analysis of Lyapunov exponent for the backward continued fraction map

Birkhoff normalization and superintegrability of Hamiltonian systems

Dynamics of the heat semigroup on symmetric spaces

Ruelle operator theorem for non-expansive systems

Complexity and cohomology for cut-and-projection tilings

Open maps between shift spaces

Rigidity of the Weyl chamber flow, and vanishing theorems of Matsushima and Weil

Basins of measures on inverse limit spaces for the induced homeomorphism

Topological properties of full groups

A renormalization group approach to quasiperiodic motion with Brjuno frequencies

A quantitative mean ergodic theorem for uniformly convex Banach spaces

Multiple polynomial correlation sequences and nilsequences

On $t$-conformal measures and Hausdorff dimension for a family of non-uniformly hyperbolic horseshoes

Measure of the Julia set of the Feigenbaum map with infinite criticality

Strong bounded operators for ergodic averages and Lebesgue derivatives

Vertices of Mather's beta function, II

Transitivity of Euclidean-type extensions of hyperbolic systems

The Mañé-Conze-Guivarc'h lemma for intermittent maps of the circle

Iteration of certain meromorphic functions with unbounded singular values

Symbolic image and invariant measures of dynamical systems

A family of 2-graphs arising from two-dimensional subshifts

Enveloping semigroups of unipotent affine transformations of the torus

Equicontinuous geodesic flows

Rates of divergence of non-conventional ergodic averages 
Rayskin, V.

Rempe, L.

Sadovskaya, V.

San Martin, L. A. B. and Seco, L. Schmidt, K. and Vershik, A.

Sumi, H. and Urbański, M.

Sun, P.

Thomsen, K.

Towsner, $\mathrm{H}$.

Tsukamoto, M.

Wang, X. and Zhang, G.

Yarmola, T.

Yuri, M.

Zaïmi, T.

Zhang, $X$.

Zhu, Y., Li, Z. and Li, X.
Theorem of Sternberg-Chen modulo the central manifold for Banach spaces

The escaping set of the exponential

Dimensional characteristics of invariant measures for circle diffeomorphisms

Morse and Lyapunov spectra and dynamics on flag bundles Algebraic polymorphisms - ERRATUM

Real analyticity of Hausdorff dimension for expanding rational semigroups

Zero entropy invariant measures for skew product diffeomorphisms

The homoclinic and heteroclinic $C^{*}$-algebras of a generalized one-dimensional solenoid

Convergence of diagonal ergodic averages

Deformation of Brody curves and mean dimension

Constructing ergodic exponential maps with dense post-singular orbits

Degenerate random perturbations of Anosov diffeomorphisms

Entropy production at weak Gibbs measures and a generalized variational principle

On numbers having finite beta-expansions

Embedding diffeomorphisms in flows in Banach spaces

Preimage pressure for random transformations 


\section{INSTRUCTIONS FOR CONTRIBUTORS}

\section{Editorial Policy}

The journal welcomes high quality contributions on topics closely related to dynamical systems and ergodic theory. Submissions in the field of differential geometry, number theory, operator algebra, differential, topological, symbolic, measurable dynamics and celestial and statistical mechanics are especially welcome. Expository survey papers and reviews of relevant books will be published from time to time.

\section{Submission of typescripts}

Two copies of the manuscript should be submitted to an executive or managing editor whose interest is closest to the material of their article. In case of doubt authors may send manuscripts to the Managing Editors at the University of Warwick. A paper may also be submitted electronically in postscript form as an attachment to an email, i.e. not in the body of an email. Please also send the $\mathrm{T}_{\mathrm{E}} \mathrm{X}$ file. If you are unable to do this, please submit the manuscript in printed form. The editor in charge of the paper will acknowledge receipt of the paper. It is important that authors inform the editor of any changes of postal and/or e.mail address while their paper is under consideration.

The editor will send the manuscript to the referee electronically, if possible, and also airmail a copy to him/her. However, the editor can decide if the material is inappropriate for the journal and will also make the final decision regarding acceptance or rejection. Should you need further information you should communicate in the first instance with the editor in charge of your paper. In an attempt to decrease the journal's backlog an editor may decline a submission (without using the normal refereeing process) if he/she considers that a 'reasonable' referee might not recommend the paper sufficiently strongly, or (possibly) if the paper is more than 30 pages long. Such summary action, with its inherent risk of unfairness, seems preferable to introducing a moratorium (the only other alternative) and has the advantage that delayed negative decisions might be avoided. This policy will be reviewed at a later date.

Submission of a paper is taken to imply that it has not been previously published and that it is not being considered for publication elsewhere. Authors of articles published in the journal assign copyright to Cambridge University Press (with certain rights reserved) and you will receive a copyright assignment form for signature on acceptance of your paper.

The journal strongly recommends submission of accepted papers in $\mathrm{ET}_{\mathrm{E}} \mathrm{X}$ using the ETDS LTTEX class file. Papers that use this class file will be processed more efficiently. A IATEX2e file etds.cls is available via anonymous ftp from the Cambridge University Press site at ftp.cup.cam.ac.uk in the directory /pub/texarchive/journals/latex/etds-cls/. In case of difficulties with these files, please contact etds@ @ sunrise-setting.co.uk or the Journal editorial office at etds@ maths.warwick.ac.uk. Alternatively, authors may use "article" style.

On final acceptance of a paper, authors should send the $\mathrm{LT}_{\mathrm{E}} \mathrm{X}$ source code on disc including the figures (line figures only) and all author-defined macro and style files, to the Managing Editors, together with a hard copy produced using the same file. Discs should be in Apple Mac or PC format and will not be returned. The publisher reserves the right to typeset any article by conventional means if the author's $\mathrm{T}_{\mathrm{E}} \mathrm{X}$ code presents problems in production.

Typescript

Papers should be typed, double-spaced, on one side only and with generous margins. The pages must be numbered.

The first page should give the title, the author's name and institution, and a short abstract intelligible to mathematicians.

The title, while brief, must be informative (e.g. A new proof of the ergodic theorem, whereas Some applications of a theorem of Birkhoff would be useless).

\section{Notation}

It is important that mathematician's expressions are clear to a printer (who is not a mathematician). For instance, $n_{k}(n$ sub $k)$ is common usage, but avoid if possible using $c \operatorname{sub} n \operatorname{sub} k$. Fractions are generally best expressed by a solidus. Complicated exponents like $\exp \left\{z^{2} \sin \theta /\left(1+y^{2}\right)\right\}$ should be shown in this and no other way.

In the typescript, italics, small capitals and capitals are specified by single, double and triple underlining. Bold-faced type is shown by wavy underlining.

It helps if displayed equations or statements which will be quoted later are numbered in order on the right of their line. They can then be referred to by, for example, 'from (7)'.

The author must enable the printer (if necessary by pencilled notes in the margin) to distinguish between similar symbols such as $o, O, \mathrm{o}, \mathrm{O}, 0 ; x, \mathrm{X}, \times ; \phi, \Phi, \varnothing ; 1,1 ; \varepsilon, \in ; \kappa, k$.

There is no need to underline Greek or script letters provided these are clearly typed. Any special symbols should be explained on a separate sheet of directions for the printer.

If an author wishes to mark the end of the proof of a theorem, the sign $\square$ may be used.

Footnotes should be avoided.

\section{Diagrams}

Figures and drawings should be on separate sheets in black ink or produced by computer to comparable quality. Photocopies are acceptable only if they are as clear as the originals. Symbols, legends and captions should be given on a transparent overlay. Each text figure must be numbered as Figure 1, Figure 2, .. and its intended position clearly indicated in the typescript. The author's name in pencil must be on all separate sheets of diagrams.

Figures should be used sparingly and only when they greatly clarify the exposition.

Tables

Tables should be numbered (above the table) and set out on separate sheets. Indicate the position of each in the text as for figures.

References

References should be collected at the end of the paper numbered in alphabetical order of the author's names. A reference to a book should give the title, in italics, and then in roman type the publisher's name and the place and year of publication:

[4] N. Dunford and J. T. Schwartz. Linear Operators. Part I. Wiley, New York, 1958.

A reference to a paper should give in italics the title of the periodical, the number of the volume and year, and the beginning and end pages of the paper. Titles should be abbreviated as in Mathematical Reviews:

[6] J. E. Littlewood. The 'pits effect' for functions in the unit circle. J. Analyse Math. 23 (1970), 236-268.

Proofs

Authors receive one set of proofs for correction. If excessive alterations to the original manuscript are requested after the paper has been typeset, the author will be charged the cost of resetting. For papers with more than one author the proofs are sent to the first named author unless the editor receives other instructions. It is important that proofs are corrected and returned promptly.

Offprints

50 offprints of each article will be supplied free to each first named author. Extra offprints may be purchased from the publisher if ordered at proof stage. 


\title{
Ergodic theory and dynamical systems
}

\author{
VOLUME 29 PART 3 JUNE 2009
}

\section{CONTENTS}

Balibrea, F., Downarowicz, T., Hric, R., Snoha, L'. and Špitalský, V. Almost totally disconnected minimal systems

Boissy, C. and Lanneau, E. Dynamics and geometry of the Rauzy-Veech induction for quadratic differentials

Bonatti, C. and Guelman, N. Transitive Anosov flows and Axiom-A diffeomorphisms

Chierchia, L. and Pusateri, F. Analytic Lagrangian tori for the planetary many-body problem

Curry, C. P., Mayer, J. C., Meddaugh, J. and Rogers Jr, J. T. Any counterexample to Makienko's conjecture is an indecomposable continuum

Feng, D.-J. and Shu, L. Multifractal analysis for disintegrations of Gibbs measures and conditional Birkhoff averages

Gelfert, K. and Rams, M. The Lyapunov spectrum of some parabolic systems

Käenmäki, A. and Shmerkin, P. Overlapping self-affine sets of Kakeya type

Jiménez López, V. and Peralta-Salas, D. Global attractors of analytic plane flows

Monteil, T. Finite blocking property versus pure periodicity

Savinien, J. and Bellissard, J. A spectral sequence for the $K$-theory of tiling spaces

Törnquist, A. Conjugacy, orbit equivalence and classification of measurepreserving group actions

Papers to appear in forthcoming issues 